\title{
Reconstruction of a long defect of the median nerve with a free nerve conduit flap
}

\author{
Andrea Campodonico, Pier Paolo Pangrazi, Francesco De Francesco, Michele Riccio \\ Department of Reconstructive Surgery and Hand Surgery, AOU Ospedali Riuniti di Ancona, Ancona, Italy
}

\begin{abstract}
Upper limb nerve damage is a common condition, and evidence suggests that functional recovery may be limited following peripheral nerve repair in cases of delayed reconstruction or reconstruction of long nerve defects. A 26-year-old man presented with traumatic injury from a wide, blunt wound of the right forearm caused by broken glass, with soft tissue loss, complete transection of the radial and ulnar arteries, and a large median nerve gap. The patient underwent debridement and subsequent surgery with a microsurgical free radial fasciocutaneous flap to provide a direct blood supply to the hand; the cephalic vein within the flap was employed as a venous vascularized chamber to wrap the sural nerve graft and to repair the wide gap $(14 \mathrm{~cm})$ in the median nerve. During the postoperative period, the patient followed an intensive rehabilitation program and was monitored for functional performance over 5 years of follow-up. Our assessment demonstrated skin tropism and sufficient muscle power to act against strong resistance (M5) in the muscles previously affected by paralysis, as well as a good localization of stimuli in the median nerve region and an imperfect recovery of two-point discrimination (S3+). We propose a novel and efficient procedure to repair $>10-\mathrm{cm}$ peripheral nerve gap injuries related to upper limb trauma.
\end{abstract}

Keywords Nerve injuries / Free flap / Nerve graft / Nerve conduit flap / Complex trauma

\author{
Correspondence: \\ Francesco De Francesco \\ Department of Reconstructive Surgery \\ and Hand Surgery, AOU Ospedali \\ Riuniti di Ancona, via Conca 71, \\ Ancona 60123, Italy \\ Tel: $+39-71-5963945$ \\ Fax: +39-71-5965297 \\ E-mail: fran.defr@libero.it
}

Received: May 27, 2019 • Revised: October 9, $2019 \bullet$ Accepted: October 18, 2019

pISSN: 2234-6163・ elSSN: 2234-6171 • https://doi.org/10.5999/aps.2019.00654 • Arch Plast Surg 2020;47:187-193

\section{INTRODUCTION}

Reconstructive surgery is undoubtedly a complicated intervention for the repair of extensive $(>6 \mathrm{~cm})$ peripheral nerve damage. In 1972, Millesi et al. [1] introduced the fascicular graft technique, which was a major improvement on autologous nerve grafts and a turning point in the ability of surgical treatment for this type of lesion to yield optimal outcomes. However, while this technique was feasible in cases of limited nerve substance loss, it could not ensure reliable reinnervation of the target organ across gaps exceeding $6 \mathrm{~cm}$. Moreover, such nerve grafts passively support axon regeneration, but do not directly promote and modulate growth [2].

Over past decades, the technique of tubulisation was proposed to address these shortcomings. In this technique, a conduit of biological or artificial nature (consisting of a vein, mesothelial chambers, silicone conduits, Maxon suture material [Covidien, Minneapolis, MN, USA], collagen, or polyglycolic acid) is interposed between the two nerve stumps [3]. This conduit provides the regenerating axon with a channel for protection and guidance, facilitating regeneration. The vein-muscle combination is currently the treatment of choice, as has been confirmed via experimental and clinical studies $[4,5]$ illustrating the regenerative potential of the vein and the ability of the muscle to pre- 
vent vein collapse and promote axon reconstruction. The veinmuscle procedure appears to be suitable for sensory nerves with gaps of $3 \mathrm{~cm}$ or less, but it is less efficient in the repair of larger nerve gaps $[6,7]$. Ample evidence supports the advantages of conventional nerve flaps over nerve grafts for bridging large nerve gaps, since nerve flaps possess sufficient blood supply at the time of nerve transfer and are associated with reduced risk of central necrosis, fibrosis, and disordered histological findings. Townsend and Taylor (1984) proposed the novel arterialized neurovenous flap to transfer nerve segments with an exclusively venous pedicle $[8,9]$. In light of this information, we propose an innovative method using a vascularized autologous vein to provide support for nerve regeneration. In this study, we report the use of a free nerve conduit flap for successful reconstruction of a peripheral nerve injury with a $>10-\mathrm{cm}$ gap following severe trauma of the extremity. The injury involved considerable substance loss of the volar surface of the forearm and indirect vascularization of the hand due to traumatic radial and ulnar artery occlusion, as well as a median nerve gap exceeding $14 \mathrm{~cm}$.

This report describes the delayed reconstruction of a long nervous defect $(>10 \mathrm{~cm})$ with considerable substance loss using a technique we termed a nerve conduit flap. The goal of this report is to demonstrate: (1) the reconstruction of a long defect of the median nerve using an autologous nerve graft vascularized and protected by the cephalic vein; (2) the reconstruction of a defect with extensive soft tissue loss; and (3) the details of how this reconstruction was performed in the context of a single surgical intervention.

\section{CASE}

A 26-year-old man from Albania presented with a broken glass injury to the proximal third of the volar surface of the right forearm. The patient had initially been referred to a hospital for treatment and underwent muscle and skin surgery with 3-0 Vicryl mattress sutures (Fig. 1), but was admitted 2 weeks later to our reconstructive and hand surgery clinic as an outpatient. This investigation was performed in compliance with the Declaration of Helsinki and the Guidelines for Good Clinical Practice. The patient provided written informed consent both for surgery and for follow-up. The follow-up study protocol was approved by the Ethics Committee (AG_1). Information regarding the initial procedure (including the operative report from the outside hospital) was unavailable.

A physical examination revealed abundant purulent secretions from the wound. The patient reported tingling and numbness in the median nerve distribution and displayed a flexion motor deficit of the long fingers and the opponens pollicis. The patient

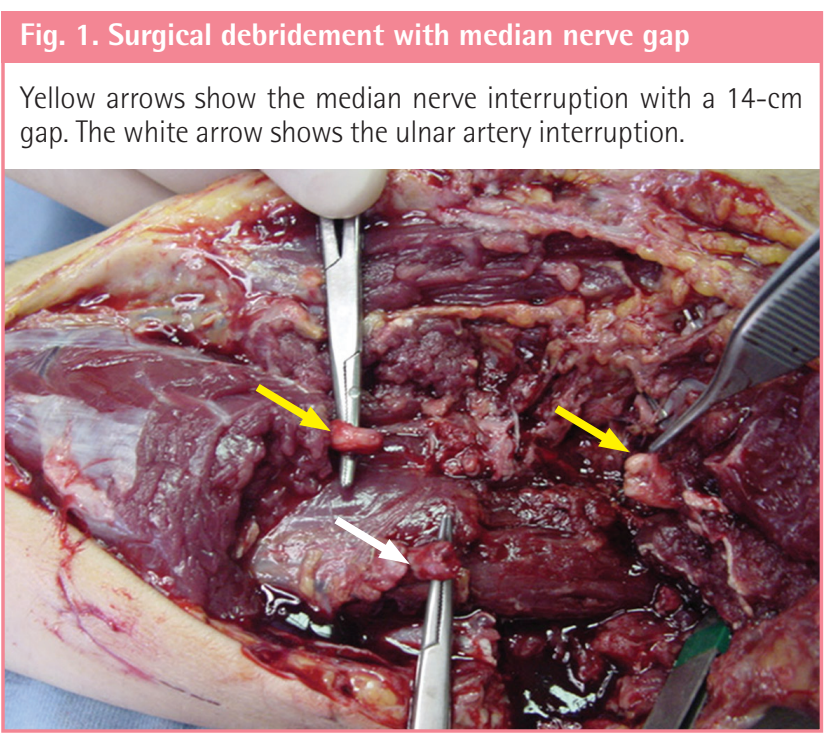

also presented with median palsy and was unable to abduct and oppose the thumb (ape hand deformity), indicative of a lesion of the main trunk of the median nerve proximal to the emergence of the anterior interosseous nerve branch. He reported sensory loss in the thumbs, index fingers, long fingers, and the radial aspect of the ring finger with impaired pronation of the forearm (due to involvement of the pronator branch) and difficulty flexing the wrist and fingers at the metacarpophalangeal and proximal interphalangeal joints. Difficulty flexing the wrist along the main axis of the forearm has been clinically attributed to a deficit of the radial carpus flexor. However, no tendon contraction was evident in this area; instead, wrist flexion along the ulnar axis was present and normal, with contraction of the relative tendon of the ulnar carpus flexor.

Clinical assessment remains the most important step in the diagnosis of nerve injury or other nerve conditions, as detailed in our previous review [2]. We assessed motor function using the British Medical Research Council scale, modified by Birch et al. [10] and proposed by Highet, and we assessed strength using a JAMAR dynamometer (JLW Instruments, Chicago, IL, USA) [11]. We determined sensory function using a Visual Analogue Scale (VAS) and tactile two-point discrimination using the test proposed by Dellon [12], which built on the work of Weber. Finally, the Nine-Hole Peg Test [13] and the Frenchay Arm Test [14] were conducted to assess motor function (Table 1). At T0 (first evaluation), we observed a strength of M0/M1, with a perceptible contraction in proximal muscles and a total active range of motion (or total active motion) of less than $20 \%$ for motor function. We also observed a result of S0 with no recovery of sensibility in the autonomous zone of the median nerve. The two-point discrimination test yielded a result of $15 \mathrm{~mm}$. Electroneuromyography (ENMG) revealed chronic median neurot- 
Table 1. Patient assessment

\begin{tabular}{|c|c|c|c|c|c|c|}
\hline Test & T0 & T1 & $\mathrm{T} 2$ & T3 & T4 & T5-7 \\
\hline $\begin{array}{l}\text { Medical Research Council (\%, compared to healthy } \\
\text { hand function) }\end{array}$ & 20 & 30 & 50 & 60 & 80 & 80 \\
\hline $\begin{array}{l}\text { JAMAR dynamometry test (\%, compared to healthy } \\
\text { hand function) }\end{array}$ & 10 & 10 & 40 & 60 & 80 & 80 \\
\hline Thumb opposition movement & Absent & Absent & Weak & Weak & Normal & Normal \\
\hline Frenchay Arm Test & 0 & 0 & 0 & 5 & 5 & 5 \\
\hline Nine-Hole Peg Test & $\begin{array}{c}\text { Subject was } \\
\text { unable to perform } \\
\text { the task }\end{array}$ & $\begin{array}{c}\text { Subject was } \\
\text { unable to perform } \\
\text { the task }\end{array}$ & $\begin{array}{l}\text { Task completed in } \\
120 \text { sec }\end{array}$ & $\begin{array}{l}\text { Task completed in } \\
70 \mathrm{sec}\end{array}$ & $\begin{array}{l}\text { Task completed in } \\
50 \mathrm{sec}\end{array}$ & $\begin{array}{l}\text { Task completed in } \\
50 \mathrm{sec}\end{array}$ \\
\hline Visual Analogue Scale sensitivity & 0 & 0 & 0 & 0 & $5-6$ & $5-6$ \\
\hline Weber test (mm) & $>15$ & $>15$ & $>15$ & $>15$ & $13-15$ & $13-15$ \\
\hline British Medical Research, Council test/Dellon test & $\mathrm{MO} / \mathrm{SO}$ & $\mathrm{MO} / \mathrm{SO}$ & $\mathrm{M} 2 / \mathrm{S} 2+$ & M3-M4/S3 & $\mathrm{M} 4 / \mathrm{S} 3+$ & $\mathrm{M} 5 / \mathrm{S} 3+$ \\
\hline Electroneuromyography & Negative & Negative & $\begin{array}{l}\text { Positive for hand } \\
\text { reinnervation- } \\
\text { related signs }\end{array}$ & $\begin{array}{l}\text { Positive for hand } \\
\text { reinnervation- } \\
\text { related signs }\end{array}$ & $\begin{array}{l}\text { Positive for hand } \\
\text { reinnervation- } \\
\text { related signs }\end{array}$ & $\begin{array}{l}\text { Positive for hand } \\
\text { reinnervation- } \\
\text { related signs }\end{array}$ \\
\hline
\end{tabular}

mesis at the proximal forearm. The patient showed a complete disruption of the axon, Schwann cells, and endoneurium with complete motor loss and complete sensory loss. An ENMG exam provided evidence for these conditions (data not available), which were confirmed by surgical exploration during debridement (Fig. 1).

The Allen test showed poor blood flow with a cold hand and little refill. Angiography showed occlusion of the distal humerus artery at the level of the splitting of the forearm branches (the radial and ulnar artery), as well as patency of the interosseous artery, the deep palmar arch, and the digital common arteries.

Two days after our first evaluation, we surgically explored the lesion under tourniquet control. The patient presented with a volar lesion to the forearm (which had been sutured in another hospital) with exposure of muscles and tendons without visible healthy bone. We cleansed the wound and biopsied the muscle, which was found to be infected by Enterobacter sakazakii and Staphylococcus xylosus and was treated with targeted antibiotics. We proceeded with complete removal of the radial flexor carpi, along with partial removal of the superficial flexor digitorum, the pronator teres, the brachioradialis, and the flexor digitorum profundus (Fig. 1). Disruption of the median nerves and the ulnar and radial arteries provided the hand with a clearly impaired blood supply, although the hand was vascularized by the interosseous arteries of the forearm. The median nerve was observed to be affected by a 14-cm defect subsequent to debridement of the infected and fibrous tissue.

One month after the first surgical procedure, a nerve conduit flap was raised from the contralateral forearm to bridge the gap in a second surgical operation (Fig. 2) after performing the Allen test. To repair the tissue substance loss and restore direct blood flow to the hand, we used a free radial fasciocutaneous

\section{Fig. 2. Schematic image of the nerve conduit flap}

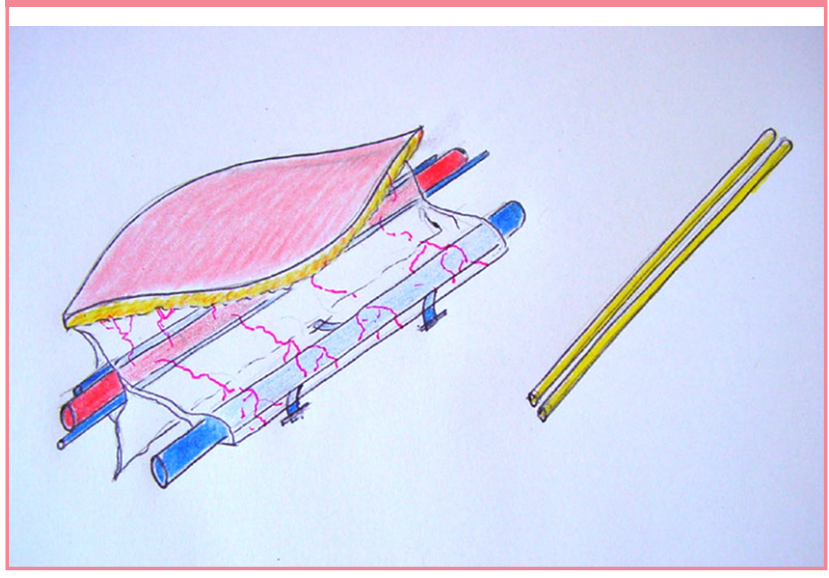

flap and performed microsurgical anastomosis to join the radial recipient artery stumps. The venae comitantes of the flap were anastomosed end-to-end to the comitantes of the humerus artery at the elbow proximally and to the radial comitantes distally. Additionally, the flap containing the cephalic vein was used as a venous vascularized chamber for the sural nerve graft and was folded in two to facilitate reconstruction of the median nerve (Fig. 3). The duplication of the sural nerve from the right leg served to increase the axon conduits in relation to the internal topography of the median nerve and was inserted into the vascularized cephalic vein. To restrict full extension, we placed a splint on the patient's hand and wrist for 30 days post-surgery. The patient was discharged 15 days after surgery and was admitted to an intensive program of rehabilitative physiokinetic therapy in which he followed a customized rehabilitation schedule. The patient was periodically monitored on an outpatient basis for 5 years; monitoring included clinical and functional examination at regular intervals to assess finger position, muscle tro- 
(A) Duplicated sural nerve inserted into the cephalic vein. (B) Venous vascularized chamber for reconstruction of the median nerve.

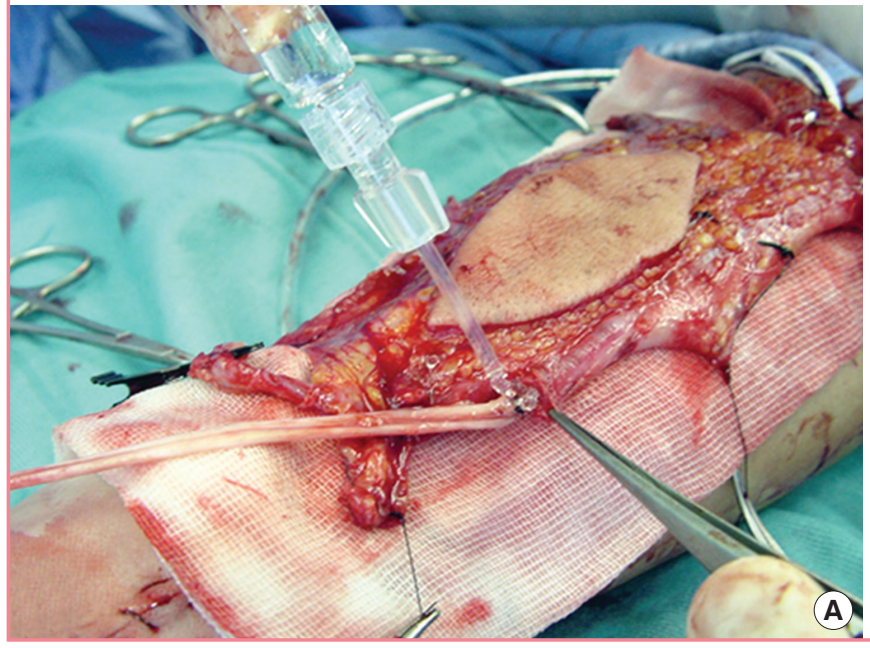

pism, articular mobility, the presence of odema and of musculotendinous retraction, and scar quality. Additionally, we assessed motor function using the British Medical Research Council scale, modified by Birch et al. [10] and proposed by Highet, and we assessed strength via a JAMAR dynamometer [11]. We determined sensory function using a VAS and tactile 2-point discrimination using the test proposed by Dellon [12], which built on the work of Weber. Finally, the Nine-Hole Peg Test [13] and the Frenchay Arm Test [14] were conducted to assess motor function.

The assessments were repeated after 2 months (T1), 6 months (T2), 12 months (T3), and 24 months (T4) and then repeated annually until 5 years post-surgery (T5, T6, and T7). ENMG was conducted as an instrumental assessment.

Periodical analysis of the results revealed steady improvement of the patient's cutaneous tropism, recovery of muscular strength, improvement of articular and tendinous mobility, and sensory recovery. With regard to cutaneous tropism, the patient improved prior to functional recovery, with improvement maintaining a positive and constant trend.

The flap progressively integrated into the recipient site while showing a steady morphological similarity to the surrounding tissues, with regression of odema and improvement of the scar; however, the scar remained hypertrophic in a section of the proximal forearm. We observed optimal outcomes in muscular strength, starting with $20 \%$ to $40 \%$ finger and wrist flexion and extension compared to the healthy extremity according to the Medical Research Council scale from T0 to T2 (Fig. 4A and B). The patient's strength steadily improved to $80 \%$ during the follow-up period. The JAMAR test results confirmed that the patient's function had increased to $70 \%$ at the last follow-up ap-

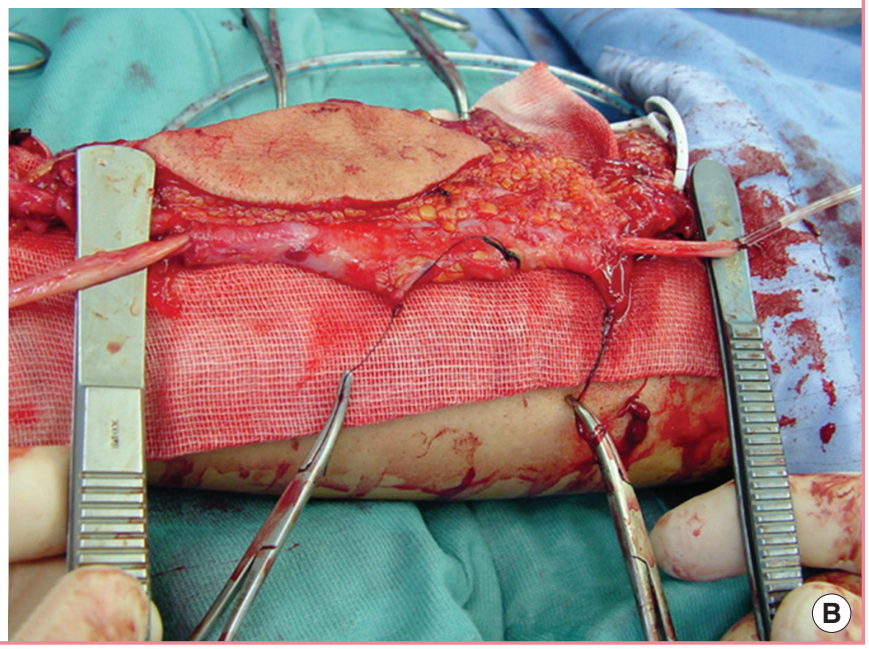

pointment, showing a delayed but constant improvement (Fig. 4C). Furthermore, we observed almost complete recovery of opponens pollicis movement, which was extremely limited during the initial postoperative period (Fig. 4D). Follow-up assessments revealed gradual and progressive improvement, reaching almost complete recovery of motor skills in terms of dexterity and agility. The Medical Research Council scale also revealed sensory recovery to a level of $\mathrm{S} 3+$ in the median nerve region, with restoration of superficial cutaneous pain and tactile sensibility and no over-response. The sensibility status of the patient, assessed with a VAS, was also noteworthy, achieving $50 \%$ to $60 \%$ fingertip sensation compared to the unimpaired extremity as well as adequate potential to sense heat, cold, and strong pressure, thus indicating satisfactory recovery of protective sensation. The two-point discriminatwwion test yielded a result of 13-15 $\mathrm{mm}$ at the final follow-up appointment (Table 1). Lastly, restoration of median nerve innervation was confirmed via ENMG, illustrating considerable improvement from the first ENMG (6 months post-surgery), which revealed complete axonotmesis (data not available). ENMG performed 1 year after surgery revealed significant reinnervation of the thenar eminence, and the final follow-up assessment displayed complete reinnervation of the hand and median nerve.

\section{DISCUSSION}

Peripheral nerve lesions associated with complex injuries of the upper limbs are frequent and are commonly associated with treatment complications in which nerve sutures are required to repair substance loss, often yielding inadequate outcomes. A traumatic injury may be too extensive for end-to-end stump su- 


\section{Fig. 4. Clinical outcomes after 5 years of follow-up}

(A) Optimal outcomes for finger and wrist flexion. (B) Optimal outcomes for finger and wrist extension. (C) Optimal outcomes for writing. (D) Complete recovery of opponens pollicis movement.
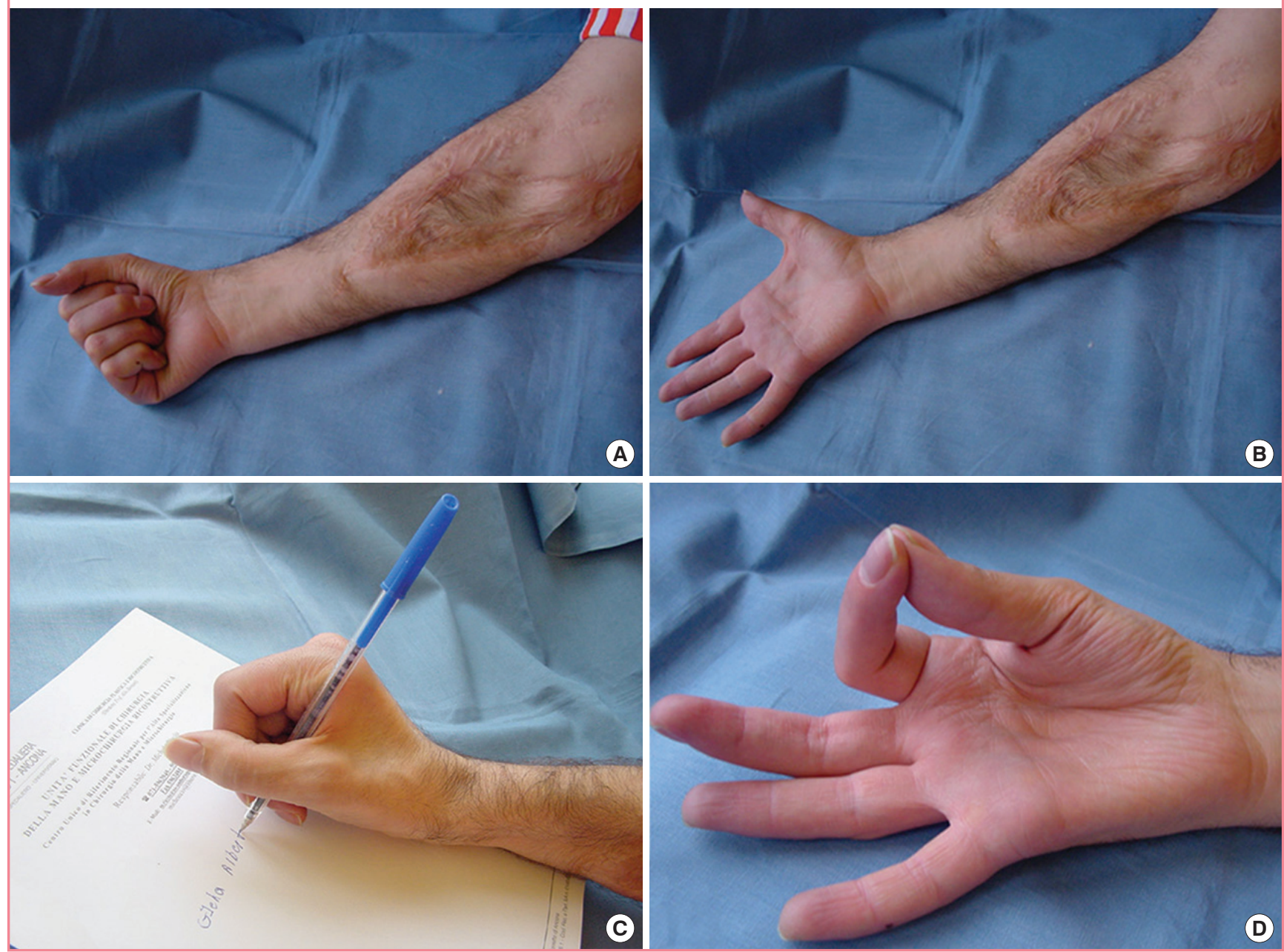

turing to be performed, although direct nerve suturing with minimal tension is required to promote satisfactory axon regeneration [6].

It is therefore clear that these circumstances require nerve grafts of various lengths, which are fundamental in the reconstructive surgery of peripheral nerves [2] to bridge the gap caused by traumatic injuries. The most accessible nerve to harvest is the sural nerve, which can be removed with minimal damage. The nerve grafts are revascularized, partially by the suture but mainly by the transfer of healthy, well-supplied, and scar-free recipient tissues. However, traditional nerve grafting has been shown to be ineffective in regeneration of the axon, with the graft undergoing ischemic degeneration and dispersion of the regenerating nerve fibers. Furthermore, traditional grafts lack substances that actively promote axon regeneration, thereby yielding poor results.

Thus, tubulisation was investigated and developed. This tech- nique uses biological or synthetic conduits such as veins, muscles, polyglycolic acid, Maxon suture material, and silicone $[2,3,7]$.

The use of muscle grafts within veins was developed $[4,5]$ following several experimental studies. Interestingly, the combination of both elements dramatically improves the outcomes, since the vein efficiently induces regeneration and the muscle prevents the vein from collapsing. Furthermore, the basal muscle membrane is rich in neurite-promoting factors such as laminin and fibronectin, which support and promote axon regeneration and Schwann cell migration [6]. The success of this combination technique can be attributed to the orientation of the regenerating axon within the biological conduit; however, it is seemingly effective only for nerve defects of $3 \mathrm{~cm}$ or less, and is ineffective for larger nerve gaps $[6,7]$. This approach prevents vein graft collapse and provides guidance [6], unlike muscle alone. In particular, muscles provide adequate adhesion for the 
growing nerve fibers provided by the basal lamina, resembling the role played by Schwann cells. These cells invade the musclein-vein conduit early and proliferate inside, mimicking the process observed in nerve grafting [15].

In complex traumatic injuries of the upper extremities, large nerve substance losses-such as the substance loss of $14 \mathrm{~cm}$ in our case- may be associated with poor blood supply of the surrounding tissues and the hands due to simultaneous lesions of the arteries, resulting in unviable, contaminated, or infected tissues that create an unsustainable recipient site.

We therefore propose a post-debridement technique to improve blood supply to the forearm and hand tissues, as well as to support the sural nerve, by inserting the sural nerve graft into a tube consisting of the vascularized cephalic vein found inside the radial flap. This is crucial for enhancing efficient axon regrowth by ensuring optimal vascularization of the graft, which promotes Schwann cell migration and the distribution of neurotropic factors and transmembrane proteins, which are essential for the modulation and regeneration of the axon fibers. Furthermore, the nerve graft remains wrapped in the cephalic vein and suspended in the blood film which flows through that vein, preventing undesirable scar adherence. This procedure supports the biological tubulisation process and promotes the supply of nutrition to the nerve graft, overcoming the current limitations in gap size and avoiding endogenous and extraneural scar adhesions. In this study, the authors present a nerve conduit flap, which contains a vein that acts as a regeneration chamber for the injured nerve to ensure both the vascularization of the autologous nerve graft and an optimal microenvironment to regenerate the nerve fibers.

The outcomes were more than satisfactory, considering the initial discrepancies and the considerable size of the median nerve gap. Improvements occurred at a relatively late stage, but this was to be expected, considering the size of the nerve gap and the area proximal to the defect. However, steady and functional recovery was observed with regard to strength, dexterity, and manual ability (M3-M4) and, to a lesser extent, to sensation (S3), which reached a satisfactory protective level in both the palm and the pulp region of the fingers. The approximate growth rate was $1.5-2 \mathrm{~mm} /$ day, illustrating ideal patterns of growth relative to nerve grafts and conventional nerve flaps in patients of the same age.

The ENMG test also confirmed effective functional recovery 12 months after surgery, along with initiation of nerve regeneration up to the thenar eminence. This illustrated that regeneration had occurred across the entire length of the graft, which exceeded $14 \mathrm{~cm}$.

This case reports the nerve conduit flap as a rapid, safe, and ef- ficient alternative for the reconstruction of a long median nerve defect after a complex traumatic injury of the upper extremities with extensive substance loss of soft tissues and a peripheral nerve gap larger than $8-10 \mathrm{~cm}$. In such cases, the use of a nerve conduit flap combined with a free flap permits microsurgical reconstruction of the tissue defect and repair of the nerve gap with excellent results in terms of functional recovery.

\section{NOTES}

\section{Conflict of interest}

No potential conflict of interest relevant to this article was reported.

\section{Ethical approval}

The study was approved by the AOU Ospedali Riuniti of Ancona Hospital (Marche Ethical Committee appoval No. CERM2019-00357) and performed in accordance with the principles of the Declaration of Helsinki. Written informed consent was obtained.

\section{Patient consent}

The patient provided written informed consent for the publication and the use of his images.

\section{Author contribution}

Conceptualization: Campodonico A. Data curation: Campodonico A, Pangrazi PP. Formal analysis: Campodonico A. Methodology: Campodonico A. Project administration: Pangrazi PP. Visualization: Pangrazi PP. Writing - original draft: Campodonico A, Pangrazi PP, De Francesco F. Writing - review \& editing: Campodonico A, De Francesco FD, Riccio M. Approval of final manuscript: all authors.

\section{ORCID}

Andrea Campodonico

https://orcid.org/0000-0003-1234-978X

Pier Paolo Pangrazi https://orcid.org/0000-0003-4886-3780

Francesco De Francesco

https://orcid.org/0000-0003-2977-7828

Michele Riccio https://orcid.org/0000-0001-5554-4759

\section{REFERENCES}

1. Millesi H, Meissl G, Berger A. The interfascicular nervegrafting of the median and ulnar nerves. J Bone Joint Surg Am 1972;54:727-50.

2. Riccio M, Marchesini A, Pugliese P, et al. Nerve repair and 
regeneration: biological tubulization limits and future perspectives. J Cell Physiol 2019;234:3362-75.

3. Patel NP, Lyon KA, Huang JH. An update-tissue engineered nerve grafts for the repair of peripheral nerve injuries. Neural Regen Res 2018;13:764-74.

4. Battiston B, Tos P, Cushway TR, et al. Nerve repair by means of vein filled with muscle grafts I. Clinical results. Microsurgery 2000;20:32-6.

5. Battiston B, Tos P, Geuna S, et al. Nerve repair by means of vein filled with muscle grafts. II. Morphological analysis of regeneration. Microsurgery 2000;20:37-41.

6. Battiston B, Tos P, Conforti LG, et al. Alternative techniques for peripheral nerve repair: conduits and end-to-side neurorrhaphy. Acta Neurochir Suppl 2007;100:43-50.

7. Riccio M, Pangrazi PP, Parodi PC, et al. The amnion muscle combined graft (AMCG) conduits: a new alternative in the repair of wide substance loss of peripheral nerves. Microsurgery 2014;34:616-22.

8. Townsend PL, Taylor GI. Vascularised nerve grafts using composite arterialized neuro-venous systems. Br J Plast Surg 1984;37:1-17.

9. D’Arpa S, Claes KEY, Stillaert F, et al. Vascularized nerve "grafts": just a graft or a worthwhile procedure. Plast Aesthet Res 2015;2:183-94.

10. Birch R, Bonney G, Winn Parry CB. Surgical disorders of peripheral nerves. London: Churchill Livingstone; 1998.

11. Bechtol CO. Grip test; the use of a dynamometer with adjustable handle spacings. J Bone Joint Surg Am 1954;36:82032.

12. Dellon AL. The moving two-point discrimination test: clinical evaluation of the quickly adapting fiber/receptor system. J Hand Surg Am 1978;3:474-81.

13. Poole JL, Burtner PA, Torres TA, et al. Measuring dexterity in children using the Nine-Hole Peg Test. J Hand Ther 2005; 18:348-51.

14. Grasel E, Biehler J, Schmidt R, et al. Intensification of the transition between inpatient neurological rehabilitation and home care of stroke patients: controlled clinical trial with follow-up assessment six months after discharge. Clin Rehabil 2005;19:725-36.

15. Geuna S, Raimondo S, Nicolino S, et al. Schwann-cell proliferation in muscle-vein combined conduits for bridging rat sciatic nerve defects. J Reconstr Microsurg 2003; 19:119-23. 